\title{
Análise de variáveis cinemáticas da corrida de jovens velocistas
}

\author{
Fernanda Stoffels \\ Ricardo S. Kober \\ Juliano Dal Pupo \\ Ivon da Rocha Junior \\ Carlos B. Mota
}

https://doi.org/10.5628/rpcd.07.01.59

\author{
U niversidade Federal de Santa M aria \\ Centro de Educação Física e D esportos \\ Laboratório de Biomecânica \\ Santa M aria - RS \\ Brasil
}

\section{RESUMO}

O objectivo deste estudo foi descrever e analisar, sob o ponto de vista cinemático, o desempenho de jovens velocistas na fase de velocidade máxima da corrida, caracterizando e comparando os valores das variáveis cinemáticas destes velocistas com atletas de nível. Fizeram parte deste estudo 7 atletas, 4 do sexo masculino e 3 do sexo feminino, com idades entre 14 e 18 anos, da cidade de Santa Maria-RS. Para a análise cinemática foi utilizado o sistema Peak Performance, Inc. utilizando 2 câmaras de alta frequência operando à $180 \mathrm{~Hz}$. As variáveis analisadas são relativas a um passo ( 2 apoios consecutivos), na fase de velocidade máxima da corrida. Cada sujeito realizou três tentativas. Os resultados encontrados mostraram similaridade das variáveis ângulo do tronco (Atron) e ângulo da coxa (Acox) com os valores encontrados na literatura para velocistas de nível mundial. No entanto, apesar de apresentarem algumas características semelhantes aos atletas de elite, os jovens velocistas estão aquém do comportamento destes em variáveis consideradas de grande importância para a performance nas corridas de velocidade, tais como amplitude e frequência de passo (Cp,Fp), distância de contacto (Dcon), oscilação vertical do CG (Ocg) e tempo da fase de suporte (Tfsup) e aérea (Tfae). Essas diferenças estão prioritariamente ligadas a factores como características físicas e nível de treinamento dos atletas, o que por sua vez pode estar associado com a idade e o tempo de prática dos jovens velocistas.

Palavras-chave: corrida de velocidade, cinemática, jovens velocistas

\section{ABSTRACT \\ Running kinematics analysis of young sprinters}

The purpose of this study was to describe the kinematics characteristics of the race of the young sprinters of Santa M aria city and region in the phase of maximum speed. The subjects of this study were 7 athletes, 4 male and 3 female, with ages ranging from 14 to 18 years. To the kinematical analysis it was used Peak Performance System, with 2 high frequency cameras operating at $180 \mathrm{~Hz}$. The movements of one stride ( 2 contacts) were analyzed in the maximum velocity phase. Each subject performed three trials. The results found show a similarity of the variables as angle of trunk and angle of limb at instant of take-off, with the values found in the literature to sprinters of world level. However, in spite of presenting some similar characteristics relatively to elite athletes, the young sprinters are below of their behavior in variables considerate of great importance to the performance in sprint running, such as, stride length and stride rate, distance of contact, vertical oscillation of CG and time of support phase and time of flight phase. Those differences may be related mainly with both physical characteristics and level of training of the athletes and could be associated with the age and time of practice of the young sprinters.

Key-words: sprint running, kinematics, young sprinters 


\section{INTRODUÇÃOO}

A destreza esportiva corrida de velocidade, aparentemente fácil de ser desempenhada, caracterizada como um movimento natural do ser humano usado para um deslocamento mais rápido no cotidiano e no esporte em geral, vem a ser um movimento relativamente complexo quando inserido na corrida atlética com um fim em si mesma (21).

Em uma abordagem mais clássica e tradicional, autores $(12,23,25)$ costumam decompor as corridas de velocidade, em especial a corrida de 100 metros rasos, em 4 fases, assim denominadas: fase de reacção; fase de aceleração; fase de velocidade máxima e a fase de desaceleração. No entanto, numa perspectiva mais actual, autores como Seagrave (24), Dick (6) identificam mais fases. O primeiro divide os $100 \mathrm{~m}$ rasos em 6 partes, desconsiderando aquecimento e acções posteriores à chegada. São elas: a saída, com as duas primeiras passadas; aceleração pura, nas 8-10 passadas seguintes; a transição, fazendo ligação com a próxima fase, a velocidade máxima; manutenção da velocidade e a parte final, caracterizada pela chegada. Em sua fase de velocidade máxima, a corrida é constituída por um conjunto de movimentos cíclicos e acentuadamente simétricos, que são os passos, considerados por Ferro ${ }^{(9)}$ a unidade básica para o estudo das corridas. Esse movimento cíclico da corrida tem duas fases principais: a fase de apoio ou suporte e a fase aérea ou suspensão (23). Um dos indicadores muito utilizado para verificar o nível técnico do velocista é a relação existente entre o tempo despendido em contacto com o solo (Tfsu) e o tempo em que o atleta está no ar (Tfae) (15), durante um passo. Para uma técnica considerada adequada, Hay (10) e Coh et al (5) apontam uma relação óptima de 60:40 em favor da fase aérea.

O atleta, durante uma corrida, deve tentar manter seu centro de gravidade em um deslocamento rectilíneo na direcção ântero-posterior, minimizando os deslocamentos em outras direcções (3). No entanto, Ferro (9) ressalta que essa translação ocorre à custa de movimentos angulares sincrónicos efectuados pelos membros superiores e inferiores. Durante a fase de apoio, o corpo do atleta avança em função da força gerada pela perna de impulso, da inércia do deslocamento, da acção de balanço da perna livre e da movimentação dos braços (3). A acção dos seg- mentos livres (membro inferior livre e membros superiores) geram momentos angulares que deverão anular-se. A acção recíproca destes segmentos, ao ser efectuada de forma adequada, garantirá uma mínima rotação do corpo durante seu deslocamento. Deve-se também ressaltar a importância da correcta disposição espacial destes segmentos no sentido de garantir a geração de um momento de inércia mínimo, importante na rapidez e economia do gesto. Os especialistas no assunto estão de acordo em que a performance alcançada nas corridas atléticas, em especial nas corridas de velocidade, deve-se às condições físicas, psíquicas e técnicas apresentadas pelos atletas, tendo esta última grande importância na performance dos mesmos. Segundo Hess apud Vonstein (28), as análises do progresso na performance de velocistas demonstram a relevância do fator técnico. Tendo em vista a importância da técnica na performance dos atletas em corridas de velocidade e sendo os conhecimentos biomecânicos, segundo Hay (10), a única base sólida e lógica para avaliá-la, torna-se importante que se invista em pesquisas desse género. Desde há algum tempo as variáveis relevantes para a performance de adultos em corridas de velocidade vêm sendo estudadas, principalmente numa abordagem mais técnico-qualitativa, mas o mesmo não ocorre tratando-se de crianças e adolescentes. Desta forma, este estudo tem como objectivo analisar, sob o ponto de vista cinemático, o desempenho de jovens velocistas na fase de velocidade máxima da corrida, caracterizando e comparando os valores das variáveis cinemáticas destes velocistas com atletas de nível. Tal estudo justifica-se à medida que trará subsídios sobre comportamento de variáveis cinemáticas de jovens velocistas, oferecendo parâmetros mais condizentes com a realidade encontrada no ambiente escolar e de iniciação desportiva, podendo assim servir de auxílio a professores e treinadores.

\section{MATERIAL E MÉTODOS}

A amostra deste estudo foi constituída por sete jovens velocistas, sendo 4 do sexo masculino e 3 do sexo feminino, com idades compreendidas entre $14 \mathrm{e}$ 18 anos, da cidade de Santa Maria - RS e região. Os sujeitos masculinos deste estudo apresentaram a estatura média de $175,00 \pm 5,40 \mathrm{~cm}$ e a média da massa corporal de $69,90 \pm 8,69 \mathrm{~kg}$. Os sujeitos femi- 
ninos apresentaram estatura média de 157,70 $\pm 5,06$ $\mathrm{cm}$ e massa corporal média de $51,70 \pm 6,00 \mathrm{~kg}$. A selecção da amostra foi intencional e os critérios para selecção foram os melhores índices em campeonatos municipais e estaduais e a frequência de no mínimo duas sessões semanais de treinamento. Para a realização da videografia tridimensional, utilizou-se duas câmeras de vídeo do sistema de análise do movimento Peak Performance Inc., operando na frequência de aquisição de imagens de $180 \mathrm{~Hz}$. A colecta foi realizada na pista atlética do Centro de Educação Física e Desportos (CEFD) da Universidade Federal de Santa Maria (UFSM), em sessão específica para esse fim. Os sujeitos foram instruídos a passar correndo em máxima velocidade pelas câmeras que estavam dispostas em diagonal ao sector em que estavam sendo realizadas as corridas (Figura 1). Os sujeitos masculinos percorreram uma distância de $40 \mathrm{~m}$ e os sujeitos femininos uma distância de $30 \mathrm{~m}$, partindo da posição em pé. Visto que os sujeitos menos velozes atingem sua velocidade máxima em um percurso antes que os mais velozes (23), deste modo, os sujeitos femininos percorreram uma distância menor. Cada sujeito realizou 3 tentativas, seguindo a mesma sequência de saída para garantir uma recuperação física igual a todos. O modelo espacial construído para a determinação do centro de gravidade foi constituído pelos seguintes segmentos: cabeça, tronco, braços direito e esquerdo, antebraço + mão direito e esquerdo, coxa esquerda e direita, perna direita e esquerda e pé direito e esquerdo. Os pontos anatómicos de referência foram os seguintes: centro de massa da cabeça; centros articulares dos ombros; centros articulares dos cotovelos; centros articulares dos punhos; centros articulares dos quadris; centros articulares dos joelhos; centros articulares dos tornozelos e extremidades anteriores dos pés. O modelo espacial encontra-se apresentado na Figura 2. Para determinar a posição do centro de gravidade foram utilizados os parâmetros inerciais, através do modelo desenvolvido por Dempster, Baumann e Galbierz citados por Riehle (20).

As imagens colectadas foram gravadas em fitas de vídeo SVHS, sendo posteriormente capturadas e transmitidas para um computador. Após isso, foram digitalizadas e passaram por um processo de recons- trução tridimensional do movimento, feito através do método DLT (Direct Linear Transformation), proposto por Abdel-Azis e Karara (1), transformando os dados bidimensionais de cada imagem em coordenadas tridimensionais, obtendo assim, parâmetros lineares e angulares. Todo esse processo foi feito pelo sistema de análise do movimento Peak Performance Inc. A digitalização foi manual e as coordenadas passaram por um processo de filtragem através do filtro de passa baixa ButtherW orth, com frequência de corte de $4 \mathrm{~Hz}$.

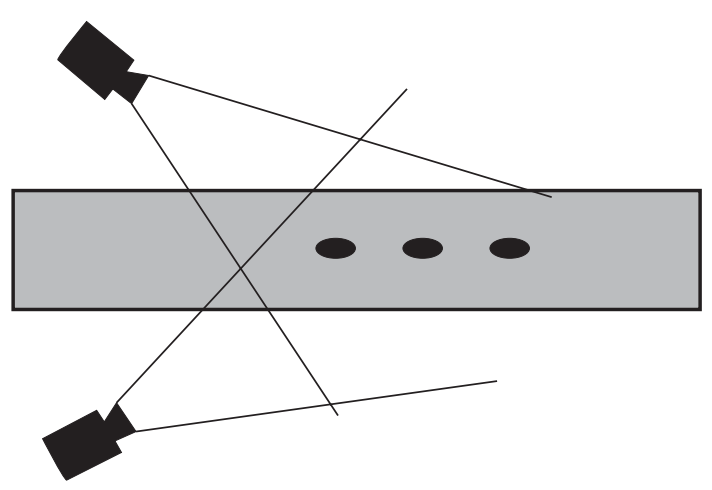

Figura 1. Posicionamento das câmeras



Figura 2. modelo espacial

Para a análise da corrida, optou-se por algumas variáveis espaciais, temporais e espaço-temporais para servir como indicadores técnicos. As variáveis foram analisadas em instantes distintos que podem 
ser visualizados na Figura 3. Estes instantes foram: instante de contacto (definido pelo quadro em que ocorre o contacto do pé com o solo); instante de máxima flexão do joelho (MFJ) e instante de impulsão (definido pelo quadro em que ocorre a perda de contacto com o solo).

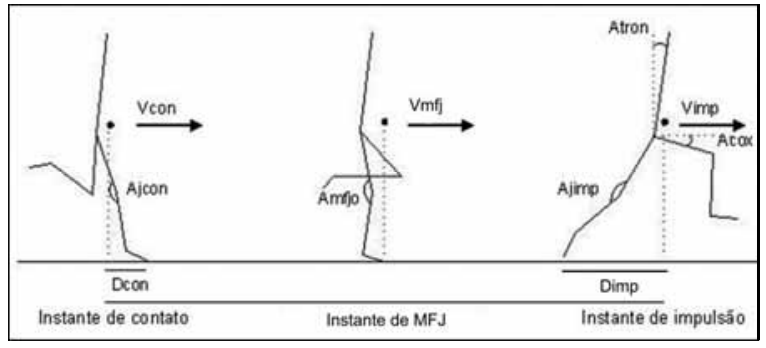

Figura 3. Instante em que as variáveis foram analisadas

A variável comprimento do passo (Cp) é considerada como a distância horizontal medida entre as pontas dos pés, projectada na direcção do movimento (eixo $\mathrm{x}$ ), em dois apoios consecutivos de pés contra-laterais. A variável distância horizontal percorrida pelo CG (DhCG) é considerada como a distância horizontal percorrida pelo CG, na direcção antero-posterior, durante as fases de apoio (iniciada ao primeiro contacto do pé com o solo e finalizada com a perda do contacto) e aérea (iniciada com a saída de um pé até o contacto do outro com o solo). A distância de contacto (Dcon) é a medida da projecção horizontal da linha entre a ponta do pé que faz o contacto e a projecção vertical do CG do sujeito no instante em que ocorre o contacto do pé com o solo. A oscilação do CG (OCG) é a medida através da diferença entre a máxima e a mínima altura do CG em relação à pista, no sentido vertical. O ângulo do tronco (Atron) indica a inclinação do tronco em relação à vertical, no sentido anterior, no instante de impulsão. $O$ ângulo da coxa livre (Acox) é o ângulo formado pela coxa livre e a horizontal, no instante de impulsão. $\mathrm{O}$ ângulo do joelho, formado pela coxa e perna de apoio, foi analisado no instante de contacto(Ajcon), no instante de máxima flexão do joelho durante o contacto(Amfj) e no instante de impulsão (Ajimp). A frequência do passo $(\mathrm{Fp})$ refere-se ao número de passos por unidade de tempo. O tempo da fase de suporte (Tfsup) é o tempo decorrido desde o contac- to do pé ao solo até a saída do mesmo pé e o tempo da fase aérea (Tfae) é o tempo decorrido desde a saída de um pé até o contacto do outro pé no solo. A velocidade média de deslocamento (Vmd), que neste estudo caracteriza a performance dos sujeitos, foi determinada como a velocidade horizontal média do CG, na direcção antero-posterior, no transcorrer de um passo. Ainda foram analisadas as velocidades do CG no instante de contacto(Vcon); instante de máxima flexão do joelho (Vmfj) e no instante de impulsão (Vimp).

Os dados obtidos foram submetidos a tratamentos estatísticos descritivos da medida de tendência central (média) e das medidas da variabilidade (desvio padrão e coeficiente de variação). As variáveis espaciais Cp, DhCG e Dcon foram normalizadas pela estatura dos sujeitos. Todos os procedimentos estatísticos foram realizados através do aplicativo Excel 2000.

\section{APRESENTAÇÃO E DISCUSSÃO DOS RESULTADOS}

As variáveis cinemáticas analisadas neste estudo encontram-se apresentadas abaixo, na Tabela 1 .

Tabela 1. Variáveis cinemáticas analisadas

\begin{tabular}{lcccccc}
\hline Variáveis & \multicolumn{3}{c}{ Sujeitos Masculinos } & \multicolumn{3}{c}{ Sujeitos Femininos } \\
& Média & DP & CV & Média & DP & CV \\
\hline Vmd (m/s) & 8,74 & 0,31 & 3,55 & 7,74 & 0,45 & 5,81 \\
Vcon (m/s) & 8,74 & 0,35 & 4,00 & 7,76 & 0,46 & 5,93 \\
Vmfj (m/s) & 8,57 & 0,33 & 3,85 & 7,57 & 0,47 & 6,21 \\
Vimp (m/s) & 8,73 & 0,34 & 3,89 & 7,73 & 0,46 & 5,95 \\
DhCG (m) & 2,04 & 0,16 & 7,84 & 1,79 & 0,07 & 3,91 \\
DhCG Norm. (\%) & 116,74 & 7,36 & 6,30 & 113,46 & 4,51 & 3,97 \\
Cp (m) & 2,06 & 0,17 & 8,25 & 1,81 & 0,06 & 3,78 \\
Cp Norm. (\%) & 117,81 & 7,97 & 6,77 & 114,85 & 3,85 & 3,35 \\
Dcon (m) & 0,36 & 0,07 & 19,44 & 0,34 & 0,04 & 11,76 \\
Dcon Norm. (m) & 20,64 & 3,76 & 18,22 & 21,48 & 3,00 & 13,97 \\
OCG (m) & 0,11 & 0,02 & 18,18 & 0,10 & 0,01 & 10,00 \\
Atron (ㅇ) & 12,43 & 2,28 & 18,34 & 14,40 & 3,53 & 24,51 \\
Acox (ㅇ) & 21,96 & 5,01 & 22,81 & 22,42 & 3,74 & 16,68 \\
Ajcon (ㅇ) & 159,12 & 7,85 & 4,93 & 148,67 & 3,97 & 2,67 \\
Amfj (ㅇ) & 147,55 & 5,27 & 3,57 & 146,00 & 4,13 & 2,83 \\
Ajimp () & 150,20 & 6,19 & 4,12 & 152,63 & 8,48 & 5,56 \\
Fp (passos/s) & 4,28 & 0,22 & 5,14 & 4,33 & 0,24 & 5,54 \\
Tfsup (s) & 0,116 & 0,01 & 8,62 & 0,127 & 0,05 & 3,94 \\
Tfae (s) & 0,117 & 0,01 & 8,55 & 0,103 & 0,01 & 9,71 \\
\hline
\end{tabular}


De acordo com Mero et al. (16), um grande número de estudos tem mostrado que, a uma velocidade constante, há um decréscimo na velocidade do CG do corpo na fase de travagem (iniciada quando o pé do corredor toca o solo e finalizada no instante em que o CG fica na vertical do apoio) e em seguida, durante a subsequente fase de propulsão (iniciada quando o CG ultrapassa o plano vertical do apoio e finalizada quando o pé abandona o solo), a velocidade aumenta. Analisando os valores médios encontrados para as variáveis Vcon, Vmfj e Vimp dos sujeitos masculinos e femininos que se encontram apresentados na Tabela 1, temos que os sujeitos masculinos apresentaram, em valores absolutos, um decréscimo da variável Vcon para a variável Vmfj de $0,18 \mathrm{~m} / \mathrm{s}$, o que corresponde ao valor percentual de $2,06 \%$, sendo que o posterior aumento da Vmfj para a Vimp foi de 0,12 $\mathrm{m} / \mathrm{s}$, ou $1,87 \%$. Já para os sujeitos femininos a queda observada foi de $0,18 \mathrm{~m} / \mathrm{s}(2,32 \%)$ da Vcon em relação à Vmfj, com posterior aumento de $0,15 \mathrm{~m} / \mathrm{s}$ $(1,98 \%)$ desta última para a Vimp. Em termos percentuais, houve, portanto, um comportamento semelhante ao apontado por Mero et al (16), que apesar de não citar valores para Vcon, encontrou uma diminuição de 1,4\% entre a Vcon e a Vmfj para velocistas femininas. Já para as variáveis Vmfj e Vimp, encontrou valores médios de $8,69 \mathrm{~m} / \mathrm{s}$ e $8,8 \mathrm{~m} / \mathrm{s}$ respectivamente, o que demonstra um aumento de aproximadamente $1,26 \%$. Mero et al apud Mero et al (16) encontraram decréscimos de $0,39 \mathrm{~m} / \mathrm{s}$ para bons velocistas, $0,43 \mathrm{~m} / \mathrm{s}$ para velocistas de nível médio e $0,53 \mathrm{~m} / \mathrm{s}$ para velocistas de nível mais baixo. No presente estudo, os jovens velocistas masculinos e femininos apresentaram um decréscimo de apenas 0,18 $\mathrm{m} / \mathrm{s}$, o que demonstraria um comportamento superior neste aspecto. No entanto, deve-se ressaltar que os valores que realmente mostram o nível de perda de velocidade e que podem ser comparados, devem ser citados na forma de percentual, isto é, relativo à velocidade do atleta. Em função disso, os $0,18 \mathrm{~m} / \mathrm{s}$ de perda de velocidade dos jovens velocistas deste estudo, representam $2,32 \%$ de perda nos sujeitos femininos, que correm a uma velocidade média de $7,76 \mathrm{~m} / \mathrm{s}$ e, apenas $2,06 \%$ nos masculinos, que correm a $8,74 \mathrm{~m} / \mathrm{s}$, demonstrando assim, comportamento superior nestes sujeitos. Deste modo, necessita-se saber a velocidade de deslocamento do atleta para realizar comparações entre distintos grupos.
O comprimento do passo pode ser verificado através da distância horizontal medida entre as pontas dos pés em dois apoios consecutivos ( $\mathrm{Cp}$ ), mais comummente utilizada na prática e relatada na literatura desportiva por ser de mais fácil mensuração ou através da distância horizontal percorrida pelo CG durante as fases apoio e aérea (DhCG). Em relação ao $\mathrm{Cp}$, constatou-se que os sujeitos masculinos deste estudo apresentaram o valor médio absoluto de 2,06 $\pm 0,17 \mathrm{~m}$ e o valor médio normalizado de $117,81 \%$. Em estudo realizado (17) na qual foram analisadas as duas melhores performances de todos os tempos até a época, na prova de 100 metros rasos masculino, Carl Lewis e Ben Johnson, nos trechos de máxima velocidade desta prova, foram encontrados o comprimento do passo absoluto para o primeiro de $2,53 \mathrm{~m}$ (valor normalizado de $134,57 \%$ ), enquanto que para o segundo o valor absoluto encontrado foi de $2,44 \mathrm{~m}$ (valor normalizado de $139,22 \%$ ), valores que se encontram bem acima daqueles encontrados neste estudo. O Cp médio absoluto apresentado pelos sujeitos femininos do presente estudo foi de 1,81 $\pm 0,06 \mathrm{~m}$ e o valor médio normalizado de $113,59 \%$. Chow ${ }^{(4)}$, realizou um estudo com 12 velocistas do sexo feminino de idades compreendidas entre os 14 e 18 anos, consistindo na realização de dois sprints sobre distância de $100 \mathrm{~m}$, tendo colectado os dados daquele em que foi obtida a melhor performance; o valor médio

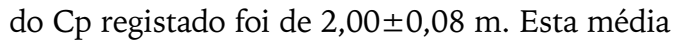
encontra-se acima dos valores encontrados para as jovens velocistas deste estudo, com a mesma faixa etária do estudo realizado por Chow ${ }^{(4)}$.

Na variável DhCG, os sujeitos masculinos deste estudo apresentaram o valor médio de 2,04 $\pm 0,16 \mathrm{~m}$ e os sujeitos femininos apresentaram o valor médio de $1,79 \pm 0,07 \mathrm{~m}$. Já para a variável $\mathrm{Cp}$, como visto anteriormente, os valores foram de 2,06 $\pm 0,17 \mathrm{~m} \mathrm{e}$ $1,81 \pm 0,06 \mathrm{~m}$ para os sujeitos masculinos e femininos, respectivamente. Percebe-se, portanto, uma pequena variação nos valores encontrados para estas duas variáveis, indicando que ambas podem ser utilizadas para verificar a amplitude da passada. Este fato está de acordo com o constatado pela literatura $(5,9)$, que equipara o comprimento das passadas $(\mathrm{Cp})$ de uma corrida de velocidade à distância percorrida pelo CG durante as fases de apoio e aérea (DhCG). Em relação à distância de contacto(Dcon), Faccioni (8) cita que deve ser mínima para que não ocorra o 
efeito de bloqueio no contacto com o solo e com isso uma diminuição da amplitude e da velocidade da passada. Este autor, juntamente com Hay (10), consideram valores abaixo de $40 \mathrm{~cm}$ ideais para esta variável. No presente estudo, os sujeitos masculinos apresentaram o valor médio de $0,36 \pm 0,07 \mathrm{~m}$ e os sujeitos femininos apresentaram valor médio de $0,34 \pm 0,04 \mathrm{~m}$. Mero e Komi (15) encontraram para os 22 sujeitos testados em seu estudo o valor médio de $0,24 \pm 0,04 \mathrm{~m}$, à máxima velocidade em um percurso de $35 \mathrm{~m}$. Confrontando-se os valores, verifica-se que os velocistas desse estudo apresentaram um comportamento inferior ao observado para atletas de outros estudos. Analisando os dados normalizados pela estatura dos sujeitos, apresentados na Tabela 1, têmse que os sujeitos femininos apresentaram um valor normalizado mais alto do que os sujeitos masculinos, o que por sua vez, pode significar um efeito de bloqueio maior, embora os valores absolutos sejam menores. A variável Dcon está directamente relacionada com o tipo de passada adoptada na corrida. Uma distância de contacto pequena pode ser a evidência de uma movimentação activa do membro inferior para trás e para baixo, o que proporcionaria uma efectividade maior durante o contacto do pé com o solo (28). Essa movimentação, denominada ciclo anterior de passada (19), é considerada uma técnica inovadora, sendo adoptada pela maioria dos atletas de alto nível, não estando claramente presente na forma de correr de nossos sujeitos. Evidenciase, pelo exposto, a necessidade de mais estudos sobre este aspecto.

O CG deve seguir uma trajectória paralela ao solo com a mínima oscilação possível (2). O mesmo autor cita ainda que quanto maior é a oscilação vertical, maior é o tempo de amortecimento e, consequentemente, menor é a velocidade, sendo que para bons atletas a diferença entre a máxima e a mínima altura do CG não supera os $5 \mathrm{~cm}$, podendo, em alguns corredores de nível mais baixo esta diferença chegar a 6 $\mathrm{cm}$. Neste estudo, os sujeitos masculinos tiveram uma oscilação média de $0,11 \pm 0,02 \mathrm{~m}$ enquanto os sujeitos femininos tiveram uma oscilação um pouco mais baixa, de 0,10 $\pm 0,01 \mathrm{~m}$. Mero et al. (16) observou a oscilação vertical do CG de 0,047 m para velocistas de melhor nível técnico, com velocidade média de $9,86 \mathrm{~m} / \mathrm{s} ; 0,050 \mathrm{~m}$ para velocistas de médio nível técnico, com velocidade média de $9,60 \mathrm{~m} / \mathrm{s}$ e 0,062 $\mathrm{m}$ para velocistas de baixo nível técnico, com velocidade média de $9,24 \mathrm{~m} / \mathrm{s}$, sendo todos velocistas masculinos. O fato dessa maior oscilação vertical do CG apresentado pelos nossos sujeitos, pode ser, em parte, explicado pelo tipo de passada predominantemente utilizada, uma vez que a maioria dos jovens velocistas analisados neste estudo não faz uso de uma técnica de passada que possibilite uma menor oscilação vertical do CG (passada tracionada, activa ou em "griffé", como a denominam os autores franceses, característica do ciclo anterior de passada). Também colabora para essa maior oscilação, o estádio técnico em que se encontram os sujeitos deste estudo, já que estão em início de carreira.

Sobre a posição do tronco, enfatiza-se que esta deve ser tal que facilite na maior medida possível os diferentes mecanismos de acção, principalmente das extremidades inferiores (11). Para Faccioni (8) a inclinação do tronco deve ser de $15^{\circ}$ ou menos quando o atleta encontra-se em grande velocidade. No estudo de Mayne (14) o ângulo de inclinação do tronco encontrado foi de $15,98 \pm 3,94^{\circ}$ para as 14 velocistas testadas. No presente estudo, os sujeitos apresentaram comportamento semelhante ao reportado pelos autores, como pode ser visualizado na Tabela 1 . Em relação ao Acox, um bom movimento de elevação da coxa livre permite ao atleta posicionar correctamente o pé para o contacto com o solo, dando início à próxima fase de apoio (10). Os valores encontrados para os sujeitos deste estudo foram de $21,96 \pm 5,01^{\circ}$ para o sexo masculino e $22,42 \pm 3,74^{\circ}$, semelhantes aos valores encontrados na literatura (26), onde foram reportados valores médios para homens de $18,40 \pm 5,70^{\circ}$ e para mulheres de $19,70 \pm 7,40^{\circ}$. Tratando-se da variável Acox, esta se relaciona directamente com a variável ângulo de inclinação anterior do tronco $(11,18,21,22)$. De acordo com os autores, seria bastante difícil o praticante conseguir uma boa elevação da coxa à frente se mantiver seu tronco com acentuada inclinação no sentido anterior. Este comportamento apontado pelos autores não foi observado para os sujeitos do presente estudo.

Em relação ao ângulo do joelho, Vonstein (28), indica valores de $165^{\circ}$ no instante de contacto, $150^{\circ}$ no de MFJ e $162^{\circ}$ no instante de impulsão. Já Vittori (27) 
aponta o valor de $140^{\circ}$ para a MFJ e $168-172^{\circ}$ no instante de impulsão. Os valores médios apresentados pelos jovens velocistas encontram-se abaixo do valor de $165^{\circ}$ apontado por Vonstein (28) no instante de contacto, como pode ser observado na Tabela 1 , indicando que os sujeitos deste estudo, principalmente os sujeitos femininos, tomaram contacto com o solo com o joelho mais flexionado do que o indicado na literatura. Já para o Amfj, os jovens velocistas apresentaram valores intermédios à àqueles propostos pelos autores acima citados $(27,28)$, enquanto que para a variável Ajimp os sujeitos do presente estudo apresentaram valores bem inferiores aos valores reportados pelos mesmos autores. Os jovens velocistas deste estudo apresentaram valores consideravelmente inferiores aos valores citados anteriormente por Vonstein (28) e Vittori (27), como pode ser observado na tabela 1 e igualmente inferiores aos valores reportados em outros estudos. Cabe salientar que neste estudo o instante de impulsão é definido como o primeiro quadro após o sujeito perder contacto com o solo, enquanto outros estudos podem se referir a este instante como o último quadro em que o sujeito ainda está em contacto com o solo. Esta pode ser uma das causas para as diferenças encontradas, visto que os sujeitos deste estudo já poderiam estar voltando a flexionar o joelho, e, por consequência, apresentarem um ângulo menor. Além disso, tais diferenças também podem ser atribuídas ao nível de desenvolvimento das capacidades físicas dos jovens velocistas, como força e flexibilidade. A magnitude da força muscular é determinante na extensão do joelho do membro inferior que gera o impulso e também no controle da acção excêntrica dos músculos durante o contacto, evitando que o joelho flexione muito.

As variáveis temporais Tfsup e Tfae referem-se ao tempo despendido para completar um passo, o qual pode ser compreendido como uma soma do tempo durante o qual o atleta está em contacto com o solo juntamente com o tempo que ele gasta no ar. $\mathrm{O}$ tempo em contacto com o solo na fase de velocidade máxima é de aproximadamente $40 \%$ do tempo total da passada (10). Estudos realizados $(2,16,17)$ em relação aos tempos despendidos para a fase de contacto e para a fase aérea concluíram que bons velocistas alcançam, quando em velocidade máxima, tempos de contacto de 0,080 s a 0,100 s e tempo da fase aérea de $0,120 \mathrm{~s}$ a $0,140 \mathrm{~s}$. No presente estudo, os sujeitos femininos apresentaram na variável Tfsup o tempo de 0,127 s $(55,21 \%)$, valor este superior ao encontrado na variável Tfae, que foi de 0,103 s (44,78\%), o que difere bastante do preconizado na literatura, evidenciando uma inversão no comportamento dos sujeitos desse estudo. Já os sujeitos masculinos apresentaram na variável Tfsup o valor de 0,116 s $(49,78 \%)$ e de 0,117 s $(50,22 \%)$ para a variável Tfae, apresentando comportamento similar ao observado em atletas de alto nível e considerado ideal pelos autores. No entanto, a similaridade caracterizou-se somente pelo fato de que a variável tempo da fase de suporte foi inferior ao tempo da fase aérea, pois os valores do Tfsup apresentados pelos sujeitos deste estudo encontraram-se bem acima dos valores propostos para atletas de alto nível. Sabendo-se que a fase aérea origina-se principalmente da propulsão gerada pelo membro de impulsão, os aspectos fisiológicos responsáveis pela produção de força, ainda pouco desenvolvidos nos jovens velocistas, podem ser os factores limitantes para a consecução de valores semelhantes aos encontrados para atletas de alto nível na relação Tfsu/Tfae. Em função disso, os valores encontrados na literatura para velocistas confirmados talvez não devessem ser considerados como parâmetros avaliativos absolutos para atletas ainda em desenvolvimento.

Em relação à $\mathrm{Fp}$, os maiores valores têm sido reportados em torno de 5 passos/s $(16,17)$. Para atletas em desenvolvimento tem-se apontado valores de 4,0 a 4,5 passos/s e 4,8 a 5,0 passos/s para os velocistas confirmados (24). O valor médio encontrado para os jovens velocistas do presente estudo foi de $4,28 \pm 0,22$ passos/s para os sujeitos masculinos e de $4,33 \pm 0,24$ passos/s para os sujeitos femininos. Estes valores assemelham-se aos valores apontados para atletas em desenvolvimento e encontram-se abaixo dos valores apontados para velocistas confirmados. A pequena diferença encontrada entre sujeitos masculinos e femininos está de acordo com Hoffmann apud Mero et al. (16), que diz não haver diferenças nesta variável em relação aos sexos, sendo a melhor performance dos sujeitos masculinos alcançada em função da maior amplitude do passo. No estudo de Chow ${ }^{(4)}$, com velocistas femininos de 14 
a 18 anos de idade, foi encontrada a frequência média do passo de 4,11 $\pm 0,19$ passos/s e no estudo de Coh et al. (5), com velocistas femininos de elite, a frequência média da passos foi de 4,33 $\pm 0,20$ passos/s. Pode-se observar que houve similaridade entre os resultados obtidos no estudo de Coh et al. (5) e o presente estudo, encontrando-se, neste último, valores da Fp maiores que os valores reportados por Chow ${ }^{(4)}$, para a mesma faixa etária.

\section{CONCLUSÕES}

Apesar de haver semelhanças entre resultados e comportamentos em algumas variáveis quando comparados com atletas de alto nível técnico, as variáveis cinemáticas da corrida que estão mais relacionadas com a performance, segundo a literatura, apresentaram-se, em sua grande maioria, com valores aquém dos reportados para atletas adultos de elite. Tais variáveis, como amplitude e frequência da passada, que têm relação directa com a velocidade foram inferiores nos jovens velocistas, bem como as variáveis Tfsup e Tfae, consideradas importantes preditores da performance pelos autores. O facto é que muitas das diferenças encontradas podem estar associadas a algumas deficiências nas capacidades condicionais como força e flexibilidade, natural em jovens com poucos anos de treinamento, mas que podem ser aprimoradas em função de um maior tempo de treinamento, como é o caso de atletas adultos. Outras variáveis que podem ser de imediato trabalhadas para reduzir a diferença verificada são algumas variáveis angulares, dependentes de ajustes no posicionamento corporal, como por exemplo, ângulo do tronco e da coxa, passíveis de serem trabalhados com exercícios específicos, em sessões técnicas.

Como sugestão para outros estudos deve-se destacar a importância de estudos próximos serem realizados utilizando-se de dados normalizados pela estatura ou comprimento do membro inferior dos sujeitos, visto que este factor poderá tornar mais fidedigna a comparação dos resultados quando se desejam informações quanto à técnica empregada pelos sujeitos. Deve-se evitar trabalhar apenas com instrumentos que predominantemente trabalhem com comparações de dados absolutos e procurar lançar mão de meios que priorizem dados relativos, como normali- zações e percentagens (proporções). Outra sugestão é que poderiam ser realizados, em estudos próximos, testes de algumas capacidades condicionais como força e flexibilidade, que são importantes para a performance nas corridas e que poderiam contribuir no entendimento dos resultados, bem como no auxílio para o treinamento.

\section{CORRESPONDÊNCIA \\ Juliano Dal Pupo}

Centro de Educação Física e Desportos - prédio 51

Laboratório de Biomecânica, sala 1007

Faixa de Camobi, km 9

Santa Maria - RS

Brasil

CEP: 97110-970

e-mail: juliano.dp@ pop.com.br 


\section{REFERÊNCIAS BIBLIOGRÁFICAS}

1. Abdel-Azis YI, Karara HM (1971). Direct Linear Transformation from Comparator Co-ordinates Into Object Space Co-ordinates. Proc. ASP/UI Symposium on Closerange Photogrammetry. Falls Church, VA.

2. Bravo J, Pascua M, Gil F, Ballesteros JM (1994). Atletismo 1: Carreras. 2ed. Madrid, Spain: Real Federación Española de Atletismo.

3. Bravo J, Pascua M, Verdugo MG, Landa LM, Gil F, Marin J (1998). A tletismo 1: Carreras y M archa. 3ed. Madrid, Spain: Real Federación Española de Atletismo.

4. Chow JW (1987). Maximum speed of female high school runners. Int J of Sport Biomech 3: p. 110-127.

5. Coh M, Dolenec A, Jost B (s/d). Kinematic, kinetic \& eletromyographic characteristics of the sprinting stride of top female sprinters. Disponível em: http://www.coachesinfo.com/article/218. Acesso em: 25 nov de 2004.

6. Dick FW (1989). Development of Maximum Sprinting speed. Reprinted from Track Technique. Disponível em: www.trackandfieldsnews.com/tecnique/109. Acesso em: 08 de maio de 2006.

7. Dyson G (1978). M ecanica del atletismo. Madrid: INEF.

8. Faccioni A (2003). Relationships between selected speed strength performance tests and temporal variables of maximal running velocity. Disponível em http://faccioni.com/reviews/thesis.pdf. Acesso em 30 jul 2004.

9. Ferro A (2001). La Carrera de Velocidad. Madrid: Editorial e Librerias Deportivas Esteban Sanz, S. L.

10. Hay JG (1981). Biomecânica das técnicas desportivas. Rio de Janeiro: Interamericana.

11. Hegedüs J (1981). Tecnicas atleticas. Buenos Aires: Stadium.

12. Letzelter M (1978). Trainings grundlagen. Rohwolt.

13. Mann R, Herman J (1985). Kinematic Analysis of Olympic Sprint Performance: Men's 200 Meters. Int J of Sports Biomech 2: 151-162.
14. Mayne CT (1994). Estudio biomecánico de la técnica de carrera lanzada en las pruebas de velocidad femenina: obtención de parámetros representativos y estudio comparativo. Rev A punts, $\mathrm{V}(\mathrm{XXXI})$ : 129 - 140.

15. Mero A, Komi V (1985). Effects of Supramaximal Velocity on Biomechanical Variables in Sprinting. Int J of Sports Biomech 1: 240 - 252.

16. Mero A, Komi PV, Gregor RJ (1992). Biomechanics of Sprint Running. Sports M ed 13(6): 376 - 392.

17. Moravec P, Ruzicka J, Susanka P (1988). Time analysis of the 100 meters events at the II World Championships in Athletics. N ew Studies in Athletics 3: $61-96$.

18. Perez CG (1978). Velocidad y Relevos. Madrid: Real Federacion Española de Atletismo.

19. Piasenta J (1988). L'Education A thletique. INSEP.

20. Riehle H (1976). Introdução na Biomecânica do Esporte. I parte. Apostila, Universidade de Konstanz.

21. Rocha Jr IC, Mota CB, Atier J, Stoffels F (2003). Validação biomecânica de uma estratégia didática para a corrida de velocidade. In: X Congresso Brasileiro de Biomecânica, O uro Preto. A nais, p. $427-430$.

22. Sant JR (1993). M etodología del A tletismo. Barcelona: Paidotribo.

23. Schmolinsky G (1982). Atletismo. Lisboa: Estampa.

24. Seagrave L (1996). Introduction to sprinting. N ew Studies in Athletics 11(2-3): 93-113.

25. Silva JF (1977). Caderno Técnico-didático - A tletismo. Brasília: Departamento de Documentação e Divulgação.

26. Tupa V, Dzhalilov A, Shulalov G (1991). Sprinting: Visual Evaluation of Technique. Legkaya Atletika 9: 70 - 73.

27. Vittori C (1997). Lallenamento del giovane corridore dai 12 ai 19 anni. Roma: Federazione Italiana di Atletica Leggera.

28. Vonstein W (1996). Some reflections on maximum speed sprinting technique. New Studies in Athletics 11(2-3): 161 165. 